\title{
Voluntary exercise prior to traumatic brain injury alters miRNA expression in the injured mouse cerebral cortex
}

\author{
W. Miao ${ }^{1}$, T.H. Bao ${ }^{1,2}$, J.H. Han ${ }^{1}$, M. Yin ${ }^{1}$, Y. Yan ${ }^{1}$, W.W. Wang ${ }^{3}$ and Y.H. Zhu \\ ${ }^{1}$ Department of Neurology, Second Affiliated Hospital, Kunming Medical University, Kunming, China \\ ${ }^{2}$ Psychiatric Hospital of Yunnan Province, Kunming, China \\ ${ }^{3}$ Department of Cardiology, Second Affiliated Hospital, Kunming Medical University, Kunming, China
}

\begin{abstract}
MicroRNAs (miRNAs) may be important mediators of the profound molecular and cellular changes that occur after traumatic brain injury (TBI). However, the changes and possible roles of miRNAs induced by voluntary exercise prior to TBI are still not known. In this report, the microarray method was used to demonstrate alterations in miRNA expression levels in the cerebral cortex of TBI mice that were pretrained on a running wheel (RW). Voluntary RW exercise prior to TBI: i) significantly decreased the mortality rate and improved the recovery of the righting reflex in TBI mice, and ii) differentially changed the levels of several miRNAs, upregulating some and downregulating others. Furthermore, we revealed global upregulation of miR-21, miR-92a, and miR-874 and downregulation of miR-138, let-7c, and miR-124 expression among the sham-non-runner, TBI-non-runner, and TBI-runner groups. Quantitative reverse transcription polymerase chain reaction data (RT-qPCR) indicated good consistency with the microarray results. Our microarraybased analysis of miRNA expression in mice cerebral cortex after TBI revealed that some miRNAs such as miR-21, miR-92a, miR874 , miR-138, let-7c, and miR-124 could be involved in the prevention and protection afforded by voluntary exercise in a TBI model.
\end{abstract}

Key words: Voluntary exercise; Running wheel; miRNA; Traumatic brain injury

\section{Introduction}

Traumatic brain injury (TBI) is a leading cause of mortality in the general U.S. population, accounting for over 50,000 deaths annually (1). Within the armed forces, however, postdeployment surveys indicate that up to $20 \%$ of soldiers who have returned from Iraq have experienced at least one TBI (2). However, there is a lack of effective therapies, and researchers are just beginning to understand the multiple cellular, molecular, and biochemical changes that contribute to outcome following TBI.

Exercise is considered beneficial for overall health of the organism during both homeostasis and disease. A growing body of evidence indicates that exercise such as wheel running or using a treadmill after the onset of spinal cord injury or TBI counteracts some of the effects of the lesion (3-5). Spontaneous exercise may be therapeutic in the management of central nervous system injury by reducing the degree of initiatory damage, increasing the number of new neurons, regulating neurogenesis, limiting the degree of secondary neuronal death, improving neuronal plasticity and cognitive function, and promoting neural repair and behavioral rehabilitation $(6,7)$.
Current research data suggest that epigenetic modifications (DNA methylation and histone acetylation) and microRNAs (miRNAs) are responsive to acute aerobic and resistance exercise (8). miRNAs are ubiquitously expressed, small, noncoding RNAs that modulate posttranscriptional gene expression. Quantifying miRNA expression levels and predicting their functions as regulators of both single targets and complex networks is technically challenging and requires a combined bioinformatics, molecular, and systems biology approach. The expression of miRNAs has also been implicated in anabolic intracellular signaling and muscle hypertrophic responses associated with resistance exercise training (9). Researchers also reported that several miRNAs are upregulated following experimentally induced TBI, using both in vivo and in vitro approaches (10). It is suggested that miRNAs may be important mediators of the profound short- and long-term molecular and cellular changes that occur after TBI. Microarray studies in animal models of $\mathrm{TBI}$ have also revealed significant changes in miRNA expression within the rodent brain after TBI (11-13).

Correspondence: Yu-hong Zhu: <yuhong_lilin@aliyun.com>.

Received April 29, 2014. Accepted December 5, 2014. First published online March 6, 2015. 
Exercise following spinal cord injury has shown promise as a means to improve functional recovery, and research suggests that the process may be mediated by miRNAs (14). However, few studies have assessed miRNA expression changes in the mouse cerebral cortex induced by TBI.

The present study used microarray analysis to measure miRNA expression levels in the cortex of TBI mice after exercise training. Mice were housed with voluntary access to a running wheel (RW) or an immobilized RW for 3 weeks before injury. The mice were then subjected to $\mathrm{TBI}$ or sham surgery. Righting reflex response and mortality following TBI were evaluated. Cerebral cortices were collected $6 \mathrm{~h}$ after TBI and subjected to miRNA analysis, and the results were confirmed using quantitative reverse transcription polymerase chain reaction (RT-qPCR) analysis.

\section{Material and Methods}

\section{Animals}

Animal use and care were in accordance with the animal care guidelines, which conformed to the Guide for the Care and Use of Laboratory Animals published by the U.S. National Institutes of Health (NIH Publication No. 85-23, revised 1996). All surgeries were performed under chloral hydrate anesthesia, and all efforts were made to minimize suffering.

\section{Groups}

Adult male C57BL/6J mice (4-4.5 months) were purchased from Kunming Medical University. Mice were housed with access to food and water ad libitum under a 12-h light/dark cycle. The mice were group-housed and allowed to acclimatize to their environment for 1 week prior to commencement of the experiments.

They were individually housed in cages equipped with a RW. The control group was exposed to an immobilized RW to provide a source of environmental enrichment. The cages were equipped with a RW (diameter $=12 \mathrm{~cm}$, width $=5 \mathrm{~cm}$; Nalge Nunc International, USA) used for voluntary exercise that rotated freely and was attached to a receiver that monitored the number of revolutions (Vital Viewer Data Acquisition System software, Mini-Mitter, USA). The mice were allowed to freely exercise in individual cages with unlimited access to the running wheel. The mean number of revolutions was calculated for each night (7:00 pm to 7:00 am, the most active period) (13). After 3 weeks, exercised animals were divided into two groups: sham operation (sham-runners) and TBI (TBI-runners). Unexercised animals were also divided into two groups: sham-non-runners and TBI-nonrunners). There were 30 mice in each group; 3 were processed for miRNA microarray analysis, and the remaining animals were used for behavior and survival analyses.
TBI

Mice were lightly anesthetized with isoflurane (i.e., until unresponsive to paw or tail pinch) and fixed on a stereotactic platform. The TBI procedure was performed as described previously (15). Briefly, the skin was treated with betadine ointment, and a midline incision was made through the scalp. A 3.5-mm circular craniotomy was made on the left parietal skull between the bregma and lambda, $0.5 \mathrm{~mm}$ lateral to the midline. The skullcap was carefully removed without disruption of the dura. The lesion was produced with a pneumatic impact device using a 3-mm diameter convex tip, mounted $20^{\circ}$ from the vertical to account for the curvature of the skull. The contact velocity was set at $4.5 \mathrm{~m} / \mathrm{s}$ with a deformation $1.5 \mathrm{~mm}$ below the dura, producing a moderately severe lesion to the cortex. Sham-operated animals were anesthetized and a craniotomy was performed, but were not subjected to head impact. After the procedure, the scalp was sutured, and each animal received a subcutaneous injection of warm physiologic saline $(1 \mathrm{~mL})$ to prevent dehydration. During surgery and subsequent recovery, body temperature was maintained with a circulating water heating pad.

\section{Recovery of righting reflex}

All mice were acclimated to the test room for 30 min after the impact or sham surgery. The mice were then placed on their backs in a clean cage. The righting reflex response was evaluated as an indicator of neurologic restoration. The time required for injured and control mice to adopt a prone position following injury or anesthesia was recorded by 3 observers blinded to the treatment. The 3 times were averaged to yield a single score for each subject.

\section{miRNA microarray}

The 7th generation of miRCURY'TM LNA Array (v. 18.0; Exiqon, Denmark) contains 3100 capture probes that cover all human, mouse, and rat miRNAs annotated in miRBase 18.0, as well as all viral miRNAs related to these species. The array also contains capture probes for 25 miRPlus $^{\mathrm{TM}}$ human miRNAs.

\section{RNA extraction}

Mice were killed by cervical dislocation and decapitated. The cerebral cortex ipsilateral to injury was removed quickly (within $60 \mathrm{~s}$ ) and frozen in $-70^{\circ} \mathrm{C}$ isopentane until processed for further analysis. After carefully rinsing in cooled phosphate-buffered saline (PBS), the tissues were homogenized on ice in TRIzol (Invitrogen, USA). Total RNA was isolated using TRIzol and an miRNeasy mini kit (QIAGEN, The Netherlands), according to the manufacturer's instructions, which efficiently recovered all RNA species, including miRNAs. RNA quality and quantity were measured using a Nanodrop spectrophotometer (ND-1000, Nanodrop Technologies, USA), and RNA integrity was determined 
by gel electrophoresis.

\section{RNA labeling}

After RNA isolation from the samples, a miRCURY'TM $\mathrm{Hy}^{\mathrm{TM}} / \mathrm{Hy}^{\mathrm{TM}}$ Power labeling kit (Exiqon, Denmark) was used according to the manufacturer's guidelines for miRNA labeling. One microgram of each sample was $3^{\prime}$ end-labeled with $\mathrm{Hy}^{\mathrm{TM}}$ fluorescent label using T4 RNA ligase and the following procedure: RNA in $2.0 \mu \mathrm{L}$ water was combined with $1.0 \mu \mathrm{L}$ CIP buffer and CIP (Exiqon). The mixture was incubated for $30 \mathrm{~min}$ at $37^{\circ} \mathrm{C}$, and the reaction was terminated by incubation for $5 \mathrm{~min}$ at $95^{\circ} \mathrm{C}$. Then $3.0 \mu \mathrm{L}$ labeling buffer, $1.5 \mu \mathrm{L}$ fluorescent label (Hy3 $\left.{ }^{\mathrm{TM}}\right), 2.0 \mu \mathrm{L}$ DMSO, and $2.0 \mu \mathrm{L}$ labeling enzyme were added into the mixture. The labeling reaction was incubated for $1 \mathrm{~h}$ at $16^{\circ} \mathrm{C}$ and was terminated by incubation for $15 \mathrm{~min}$ at $65^{\circ} \mathrm{C}$.

\section{Array hybridization}

After stopping the labeling procedure, the $\mathrm{Hy}^{\mathrm{TM}}$ labeled samples were hybridized to the miRCURY' ${ }^{\mathrm{TM}}$ LNA Array (v.18.0, Exiqon), according to the array manual. The total $25 \mu \mathrm{L}$ mixture from each of the $\mathrm{Hy}^{\mathrm{TM}}$-labeled samples, together with $25 \mu \mathrm{L}$ of hybridization buffer, was first denatured for $2 \mathrm{~min}$ at $95^{\circ} \mathrm{C}$, incubated on ice for $2 \mathrm{~min}$, and then hybridized to the microarray for $16-20 \mathrm{~h}$ at $56^{\circ} \mathrm{C}$ in a 12 -Bay Hybridization System (Hybridization System-NimbleGen Systems, Inc., USA), which provides active mixing action and a constant incubation temperature to improve hybridization uniformity and enhance the signal. Following hybridization, the slides were removed, washed several times using the Wash buffer kit (Exiqon), and finally dried by centrifugation for $5 \mathrm{~min}$ at $1000 \mathrm{~g}$. Then, the slides were scanned using the Axon GenePix 4000B microarray scanner (Axon Instruments, USA).

\section{Data analysis}

Scanned images were imported into the GenePix Pro 6.0 software (Axon Instruments) for grid alignment and data extraction. Replicated miRNAs were averaged, and miRNAs with intensities $\geqslant 30$ in all samples were selected to calculate the normalization factor. Expressed data were normalized using the median normalization. After normalization, differentially expressed miRNAs were identified through fold-change filtering. Hierarchical clustering was performed using the MEV software ( $v$ 4.6, TIGR Spotfinder, USA).

\section{miRNA expression analysis}

RT-qPCR was performed to measure miRNA levels. cDNA was synthesized from total RNA using genespecific primers according to the TaqMan MicroRNA assay protocol according to the manufacturer's protocol (Applied Biosystems, USA). RT-qPCR of miRNA was performed using an Applied Biosystems 7300 Sequence Detection system. The $10-\mu \mathrm{L}$ PCR reaction contained
$0.67 \mu \mathrm{L}$ reverse transcription product, $1 \times$ TaqMan Universal PCR master mix, and $1 \mu \mathrm{L}$ primer and probe mix, according to the TaqMan MicroRNA Assay protocol (Applied Biosystems). Samples were normalized to snoRNA202 (16). The threshold cycle was defined as the fractional cycle number at which the fluorescence exceeded the fixed threshold (17). The relative expression levels of genes were determined using the 2-delta $\mathrm{Ct}$ method.

\section{Statistical analysis}

Data are reported as means \pm SE. Kaplan-Meier survival analysis with the log-rank significance test was used to measure the mortality rates among mice with different treatments. Alterations in the recovery of righting reflex after TBI among different groups were compared using two-way ANOVA. Between- and within-group differences were tested using a repeated-measure ANOVA. Post hoc paired $t$-tests were used to assess intragroup interaction effects between specific time points when the ANOVA models produced significant main effects. Unpaired $t$-tests were used to compare baseline differences between groups. Differences were considered to be significant at $\mathrm{P} \leq 0.05$ unless otherwise indicated. All analyses were performed with the SPSS version 14 software (SPSS Inc., USA).

\section{Results}

\section{Mortality rate after TBI}

We compared mortality among the four experimental groups during a 14-day follow-up period. After 14 days of observation, 15 mice were alive in the TBI-runners group (55\% survival) but only 8 mice were alive in the TBI-nonrunners group (30\% survival). The difference in the mortality rate between these two groups was significant $(P<0.05)$, but the difference between the sham groups was not $(P>0.05$, Figure 1$)$.

\section{Recovery of righting reflex after TBI}

Mice ( $n=27 /$ group) pre-exposed to a RW or immobilized RW were exposed to the head impacts, and the time to recover the righting reflex was recorded. The main effect of injury on righting reflex recovery was significant $(P<0.05)$, as was the main effect of RW $(P<0.05$, Figure 2). RW prior to injury did not have an effect on recovery of the righting reflex in sham-operated mice $(P>0.05)$, but the righting time was shorter in TBI-runners $(P<0.05$, Figure 2).

\section{Different miRNA expression profiles in TBI-runner mice}

To identify differentially expressed miRNAs, we performed fold-change filtering on the samples in the four groups. The threshold is fold change $\geqslant 2.0$. In non-runner groups, expression levels of 109 miRNAs were different 


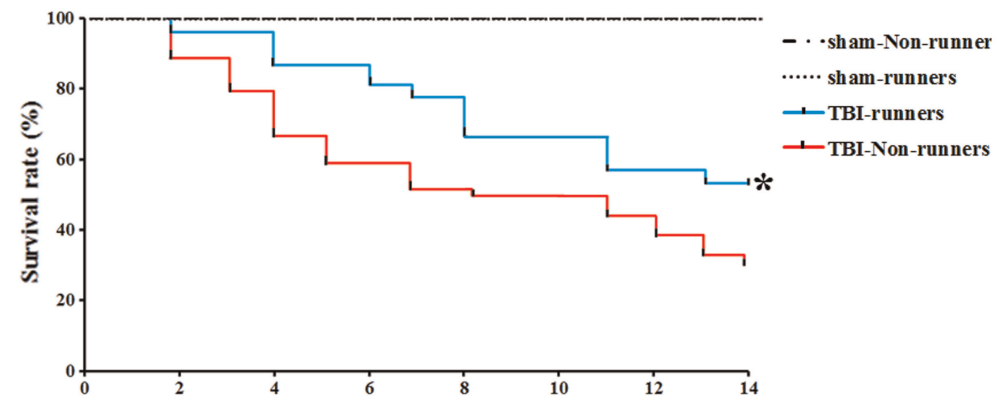

Figure 1. Percentage survival of traumatic brain injury (TBI) mice pre-treated with running wheel (RW) exercise. The number of mice pre-treated with voluntary RW that died during the 14-day postinjury follow-up period was significantly lower compared to those from injury control (TBI-nonrunner) (log-rank test, ${ }^{*}$ chi-square $=6.341, \mathrm{P}=$ 0.0278 compared to TBI-non-runner). The results indicated that pre-treatment of traumatized mice with $\mathrm{RW}$ reduced the mortality rates significantly.

between sham- and TBI-injured mice, including 64 and 45 up-and down-regulated miRNAs, respectively (Figure 3).

Effects of exercise on miRNA expression profiles in sham-operated or TBI mice were also detected. The data showed that 87 miRNAs were differently expressed between the sham-non-runner and sham-runner groups; 61 and 26 miRNAs were up- and down-regulated, respectively. We also identified 33 miRNAs that were differently modulated between the TBI-non-runner and TBI-runner groups; 20 and 13 miRNAs were up- and down-regulated, respectively (Figure 3 ).

We focused on six miRNAs (miR-21, miR-92a, miR874, miR-138, let-7c and miR-124) that were different between the sham-non-runner and TBI-non-runner groups and between the TBI-non-runner and TBI-runner groups (Figure 4).

\section{Quantitative analysis of miRNAs}

The expressions of miR-21, miR-92a, miR-138, let-7c, miR-124, and miR-874 were measured by RT-qPCR. The data showed that in the TBI-non-runner group, compared

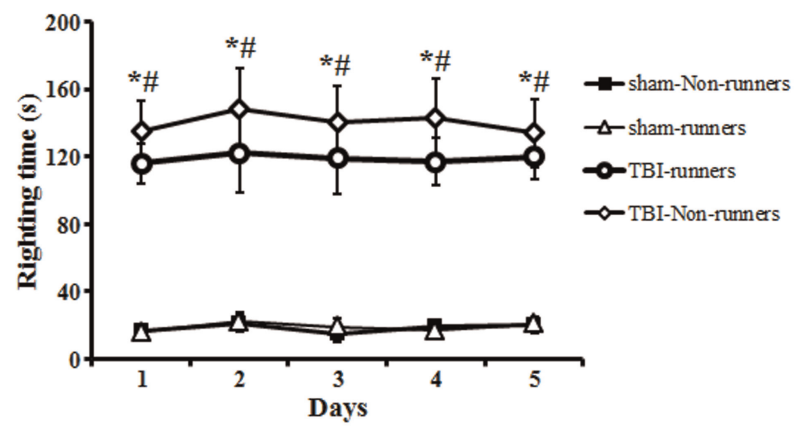

Figure 2. Recovery of righting reflex in traumatic brain injury (TBI) mice pre-trained by running wheel (RW) exercise. Controls were anesthetized and sham operated but not subjected to head injury. Results are reported as means $\pm S E$ of the time (in seconds) required for mice to right themselves after anesthesia with or without TBI. The main effect of treatment on recovery of the righting reflex was significant $(P<0.001$, two-way ANOVA). The within group effect of days was not significant for either group. ${ }^{*} \mathrm{P}<0.05$ vs TBI-runners; ${ }^{\#} \mathrm{P}<0.05$ vs sham-non-runners.

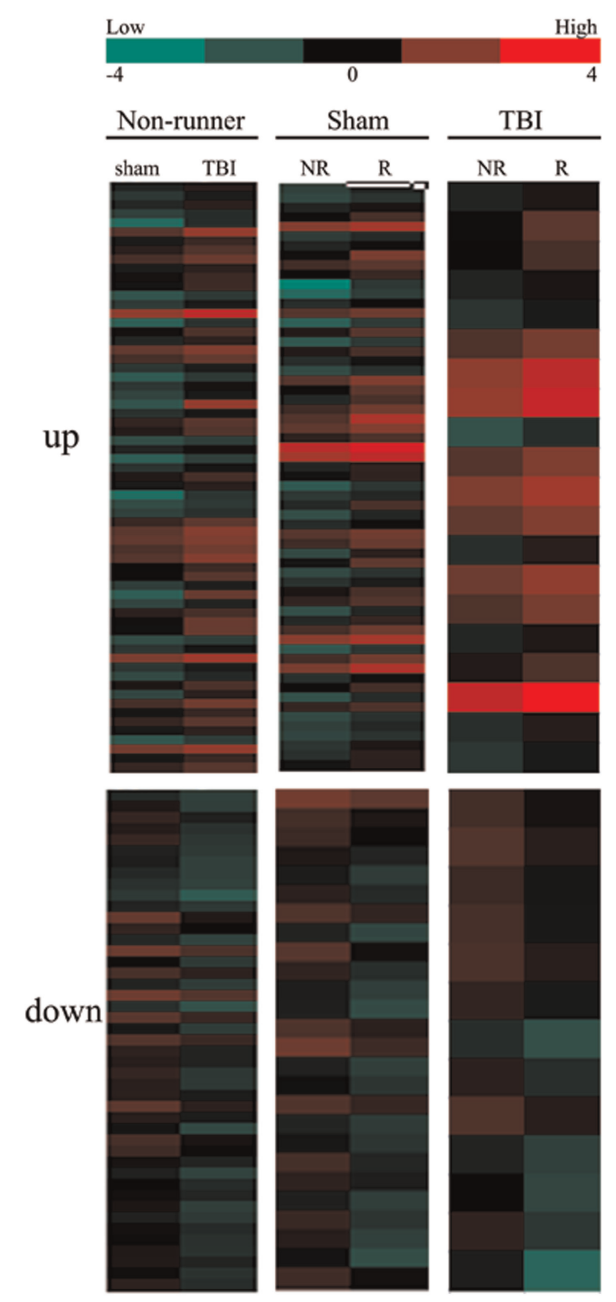

Figure 3. miRNA analysis of samples from the four groups. The heat-map shows the significant differentially expressed miRNAs. miRNA expression in paired non-runners (NR) from sham-nonrunners and traumatic brain injury (TBI)-non-runners, shamoperated mice from sham-non-runners and sham-runners $(R)$, as well as TBI mice from TBI-non-runners and TBI-runners were profiled. Red and green color scales represent high and low expression, respectively. 


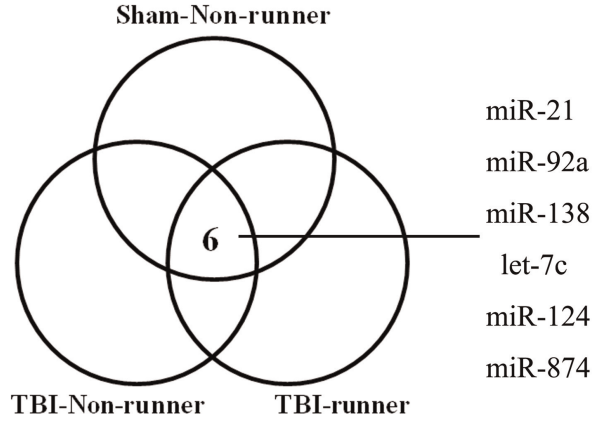

Figure 4. Differential expressions of miRNAs among sham-nonrunners, traumatic brain injury (TBI)-non-runners and $\mathrm{TBI}$ runners. The ideogram showed there were 6 miRNAs (miR-21, miR-92a, miR-874, miR-138, let-7c and miR-124), which were different between the sham-non-runners and TBI-non-runners groups, as well as between the TBI-non-runners and TBI-runners groups.

with the sham-non-runners, miR-21, miR-92a and miR874 were increased, while miR-138, let-7c and miR-124 were significantly decreased (Figure 5A, $\mathrm{P}<0.05$ ). However, in the TBI-runner group, the levels of miR-21, miR-92a, and miR-874 decreased, while those of miR138, let-7c, and miR-124 increased compared with the TBI-non-runner group (Figure 5B, $\mathrm{P}<0.05$ ).

\section{Discussion}

The present study revealed differential miRNA expression between sham and TBI mice, as well as between TBI mice pretreated with and without RW exercise. The results revealed that voluntary RW exercise prior to TBI significantly reduced the mortality rate and righting time in injured mice. Moreover, miR-21, miR-92a, miR-138, let7c, miR-124 and miR-874 were significantly altered in TBI mice that had performed RW exercise. These data indicate that miRNA modulation mediated by voluntary RW exercise might be associated with the improved outcomes of mice after TBI.

The effects of exercise on genes encoding neurotrophins and other proteins suggest that exercise could regulate anatomical changes that support brain plasticity. Others have demonstrated that spontaneous exercise might be therapeutic in the management of central nervous system injury $(5,18)$. Our findings reveal that voluntary RW exercise prior to TBI significantly reduced the mortality rate and facilitated the recovery of the righting reflex in TBI mice. We propose that an epigenetic mechanism might be involved in voluntary RW exerciseinduced amelioration of functional deficits in mice subjected to TBI.

An earlier study revealed that uphill training affected levels of circulating muscle-related miRNAs, which suggested that these miRNA could be markers or mediators of physiological adaptations (19). Microarray
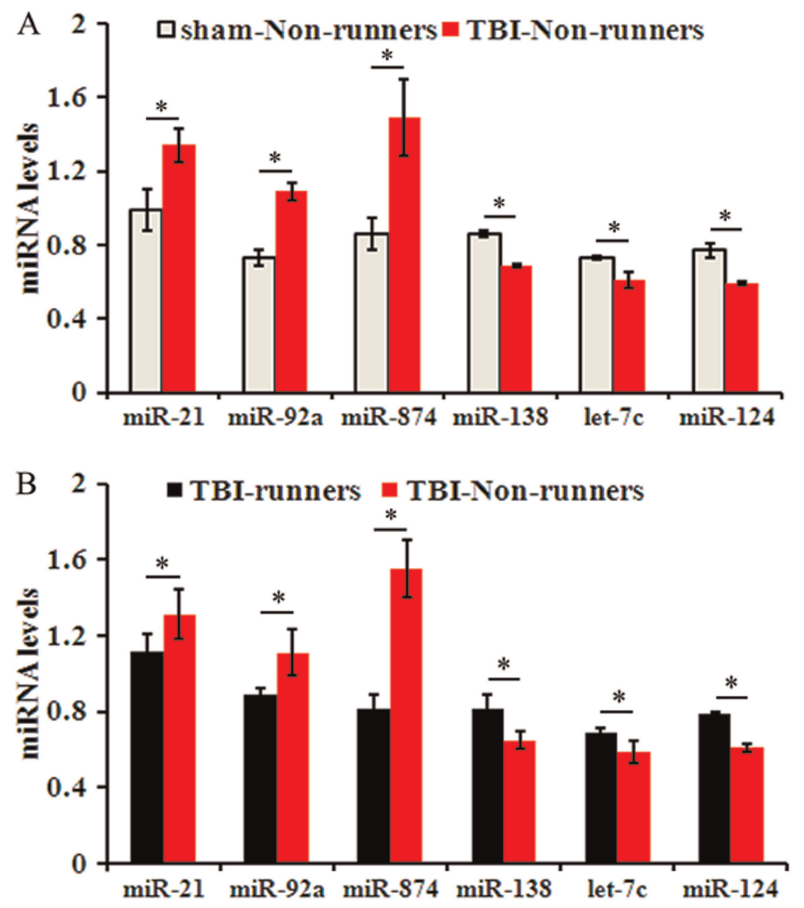

Figure 5. Expressions of miRNA detected by quantitative RTPCR. Expression levels of miR-21, miR-92a, miR-874, miR-138, let-7c and miR-124 in sham-non-runners and traumatic brain injury (TBI)-non-runners $(A)$ or in TBI-non-runners and TBIrunners group $(B)$. Data are reported as means $\pm \mathrm{SE}$. ${ }^{*} \mathrm{P}<0.05$ (Student's $t$-test).

analyses in rodent models of TBI have revealed dynamic temporal regulation of miRNA expression within the cortex and hippocampus $(11,12,20)$. Reports have identified miR-16, miR-92a, and miR-765 in the plasma of patients with TBI as promising diagnostic biomarkers in TBI (2123). However, few studies have focused on changes in miRNA expression induced by voluntary RW exercise prior to $\mathrm{TBI}$ in mice. Our findings demonstrate that previous voluntary RW exercise before TBI altered the miRNA maps in the cerebral cortex ipsilateral to injury, indicating new roles of miRNAs involved in exerciseinduced repair in a TBI model.

We observed numerous differential expressions of miRNAs between injured and sham mice and between $\mathrm{TBI}$ mice pretreated with RW and TBI mice without RW exercise. Hierarchical cluster analysis of these miRNAs demonstrated that both TBI and spontaneous RW for 3 weeks before TBI induced miRNA profiles alterations, suggesting an epigenetic mechanism that may underlie exercise-induced improvements in TBI models.

We found that miR-21, miR-92a, miR-138, let-7c, miR124, and miR-874 expression levels were different between the sham-non-runner and TBI-non-runner groups, as well as between the TBI-non-runner and TBIrunner groups. According to gene-ontology bioinformatics 
analyses, the predicted targets of these miRNAs are involved in signal transduction, transcription, proliferation, and differentiation. The miRNAs dysregulated in the chronic stages of TBI are predicted to regulate genes involved in cytoskeletal organization and intracellular trafficking-processes linked to brain repair mechanisms (24).

Reports have suggested that upregulated miR-21 expression in the hippocampus after TBI might influence multiple components of TBI pathophysiology $(20,23)$. A previous analysis of miRNA expression in rat cerebral cortex after TBI demonstrated that miR-21 might be involved in TBI pathophysiology (15). Furthermore, an earlier report demonstrated that circulating miRNA levels of miR-16, miR-92a, and miR-765 were altered after TBI, providing a rich new source of potential molecular biomarkers (23). Interestingly, overexpression of miR874 induced by TBI in vivo leads to increased vulnerability of transfected neurons (10). We found that miR-21, miR92a, and miR-874 levels increased in TBI-non-runners compared with the sham-non-runner and TBI-runner groups. Given these facts, we hypothesize that downregulation of miR-21, miR-92a, and miR-874 were involved in voluntary RW exercise-induced recovery in TBI mice.

We also observed downregulation of miR-138, let-7c and miR-124 in the cortex ipsilateral to the injured side of non-runners with TBI. miR-138 has various biological

\section{References}

1. Coronado VG, Xu L, Basavaraju SV, McGuire LC, Wald MM, Faul MD, et al. Surveillance for traumatic brain injuryrelated deaths - United States, 1997-2007. MMWR Surveill Summ 2011; 60: 1-32.

2. Shively SB, Perl DP. Traumatic brain injury, shell shock, and posttraumatic stress disorder in the military - past, present, and future. J Head Trauma Rehabil 2012; 27: 234-239, doi: 10.1097/HTR.0b013e318250e9dd.

3. Hutchinson KJ, Gomez-Pinilla F, Crowe MJ, Ying Z, Basso DM. Three exercise paradigms differentially improve sensory recovery after spinal cord contusion in rats. Brain 2004; 127: 1403-1414, doi: 10.1093/brain/awh160.

4. Ying Z, Roy RR, Zhong H, Zdunowski S, Edgerton VR, Gomez-Pinilla F. BDNF-exercise interactions in the recovery of symmetrical stepping after a cervical hemisection in rats. Neuroscience 2008; 155: 1070-1078, doi: 10.1016/ j.neuroscience.2008.06.057.

5. Vaynman S, Ying Z, Gomez-Pinilla F. Hippocampal BDNF mediates the efficacy of exercise on synaptic plasticity and cognition. Eur J Neurosci 2004; 20: 2580-2590, doi: 10.1111/j.1460-9568.2004.03720.x.

6. Griesbach GS, Hovda DA, Gomez-Pinilla F. Exerciseinduced improvement in cognitive performance after traumatic brain injury in rats is dependent on BDNF activation. Brain Res 2009; 1288: 105-115, doi: 10.1016/j.brainres. 2009.06.045.

7. Itoh T, Imano M, Nishida S, Tsubaki M, Hashimoto S, Ito A, functions and is involved in tumor progression and metastasis, cell differentiation, DNA damage, and disease; its expression is often downregulated in cancers (25). Studies have shown that let-7c miRNA plays important roles in human liver development and disease. Overexpression of let-7c miRNA represents a novel therapeutic approach for protecting human hepatocytes from oxidant injury as may occur in hepatitis and other forms of liver disease (26). However, the possible roles of miR-138 and let-7c in TBI are still unknown. The present results suggested that downregulation of $\mathrm{miR}-138$ and let7c might play protective roles, in the event of exercise taken prior to $\mathrm{TBI}$, by reducing mortality and restoring neurologic function. Recent studies have demonstrated an important role for miR-124, the most abundant and well-conserved brain-specific miRNA, in promoting neurite outgrowth and elongation during neuronal differentiation (27). Given the existing evidence, we propose that the decline of miR-124 in TBI mice without RW exercise might contribute to TBI-induced neuronal loss.

In conclusion, the present study used microarray analysis to measure miRNA expression levels in the cerebral cortex of mice with or without RW exercise before TBI. Our research revealed numerous differential expressions of miRNAs among the three groups. Specifically, miR-21, miR-92a, miR-138, let-7c, miR-124, and miR-874 could be involved in the protection induced by spontaneous RW exercise before TBI. et al. Exercise inhibits neuronal apoptosis and improves cerebral function following rat traumatic brain injury. $J$ Neural Transm 2011; 118: 1263-1272, doi: 10.1007/s0070 2-011-0629-2

8. Denham J, Marques FZ, O'Brien BJ, Charchar FJ. Exercise: putting action into our epigenome. Sports Med 2014; 44: 189-209, doi: 10.1007/s40279-013-0114-1.

9. Pasiakos SM, McClung JP. miRNA analysis for the assessment of exercise and amino acid effects on human skeletal muscle. Adv Nutr 2013; 4: 412-417, doi: 10.3945/ an.113.003699.

10. Truettner JS, Motti D, Dietrich WD. MicroRNA overexpression increases cortical neuronal vulnerability to injury. Brain Res 2013; 1533: 122-130, doi: 10.1016/j.brainres.2013.08. 011.

11. Hu Z, Yu D, Almeida-Suhett C, Tu K, Marini AM, Eiden L, et al. Expression of miRNAs and their cooperative regulation of the pathophysiology in traumatic brain injury. PLOS One 2012; 7: e39357, doi: 10.1371/journal.pone.0039357.

12. Redell JB, Liu Y, Dash PK. Traumatic brain injury alters expression of hippocampal microRNAs: potential regulators of multiple pathophysiological processes. J Neurosci Res 2009; 87: 1435-1448, doi: 10.1002/jnr.21945.

13. Bao TH, Miao W, Han JH, Yin M, Yan Y, Wang WW, et al. Spontaneous running wheel improves cognitive functions of mouse associated with miRNA expressional alteration in hippocampus following traumatic brain injury. $J \mathrm{Mol}$ 
Neurosci 2014; 54: 622-629, doi: 10.1007/s12031-0140344-1.

14. van den Brand R, Heutschi J, Barraud Q, DiGiovanna J, Bartholdi K, Huerlimann M, et al. Restoring voluntary control of locomotion after paralyzing spinal cord injury. Science 2012; 336: 1182-1185, doi: 10.1126/science.1217416.

15. Rosi S, Belarbi K, Ferguson RA, Fishman K, Obenaus A, Raber J, et al. Trauma-induced alterations in cognition and Arc expression are reduced by previous exposure to $56 \mathrm{Fe}$ irradiation. Hippocampus 2012; 22: 544-554, doi: 10.1002/ hipo. 20920.

16. Bhalala OG, Pan L, Sahni V, McGuire TL, Gruner K, Tourtellotte WG, et al. microRNA-21 regulates astrocytic response following spinal cord injury. J Neurosci 2012; 32: 17935-17947, doi: 10.1523/JNEUROSCI.3860-12.2012.

17. Ouchi $Y$, Banno Y, Shimizu Y, Ando S, Hasegawa H, Adachi $\mathrm{K}$, et al. Reduced adult hippocampal neurogenesis and working memory deficits in the Dgcr8-deficient mouse model of 22q11.2 deletion-associated schizophrenia can be rescued by IGF2. J Neurosci 2013; 33: 9408-9419, doi: 10.1523/JNEUROSCI.2700-12.2013.

18. Koo HM, Lee SM, Kim MH. Spontaneous wheel running exercise induces brain recovery via neurotrophin-3 expression following experimental traumatic brain injury in rats. $J$ Phys Ther Sci 2013; 25: 1103-1107, doi: 10.1589/jpts.25. 1103.

19. Banzet S, Chennaoui M, Girard O, Racinais S, Drogou C, Chalabi $\mathrm{H}$, et al. Changes in circulating microRNAs levels with exercise modality. J Appl Physiol 2013; 115: 12371244, doi: 10.1152/japplphysiol.00075.2013.

20. Lei P, Li Y, Chen X, Yang S, Zhang J. Microarray based analysis of microRNA expression in rat cerebral cortex after traumatic brain injury. Brain Res 2009; 1284: 191-201, doi: 10.1016/j.brainres.2009.05.074.

21. Svetlov SI, Prima V, Kirk DR, Gutierrez H, Curley KC, Hayes RL, et al. Morphologic and biochemical characterization of brain injury in a model of controlled blast overpressure exposure. J Trauma 2010; 69: 795-804, doi: 10.1097/TA.0b013e3181bbd885.

22. Papa L, Akinyi L, Liu MC, Pineda JA, Tepas JJ III, Oli MW, et al. Ubiquitin C-terminal hydrolase is a novel biomarker in humans for severe traumatic brain injury. Crit Care Med 2010; 38: 138-144, doi: 10.1097/CCM.0b013e3181b788ab.

23. Redell JB, Moore AN, Ward NH III, Hergenroeder GW, Dash PK. Human traumatic brain injury alters plasma microRNA levels. J Neurotrauma 2010; 27: 2147-2156, doi: 10.1089/neu.2010.1481.

24. Bhalala OG, Srikanth M, Kessler JA. The emerging roles of microRNAs in CNS injuries. Nat Rev Neurol 2013; 9: 328339, doi: 10.1038/nrneurol.2013.67.

25. Li J, Chen Y, Qin X, Wen J, Ding H, Xia W, et al. MiR-138 downregulates miRNA processing in HeLa cells by targeting RMND5A and decreasing Exportin-5 stability. Nucleic Acids Res 2014; 42: 458-474, doi: 10.1093/nar/gkt839.

26. Hou W, Tian Q, Steuerwald NM, Schrum LW, Bonkovsky $\mathrm{HL}$. The let-7 microRNA enhances heme oxygenase- 1 by suppressing Bach1 and attenuates oxidant injury in human hepatocytes. Biochim Biophys Acta 2012; 1819: 1113-1122, doi: 10.1016/j.bbagrm.2012.06.001.

27. Gu X, Meng S, Liu S, Jia C, Fang Y, Li S, et al. miR-124 represses ROCK1 expression to promote neurite elongation through activation of the PI3K/Akt signal pathway. $J \mathrm{Mol}$ Neurosci 2014; 52: 156-165, doi: 10.1007/s12031-0130190-6. 